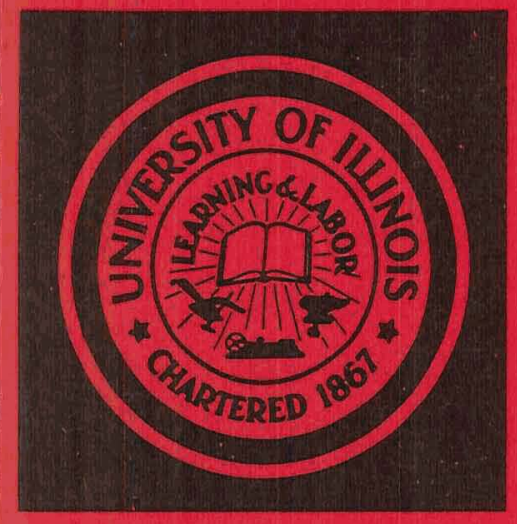

COLLEGE

$N 71-32245$

OF

ENGINEERING

UNIVERSITY OF ILLINOIS AT GHIGAGO CIRGLE

\title{
A TURBULENCE DESCRIPTION OF COUETTE FLOW
}

Paul M. Cbung

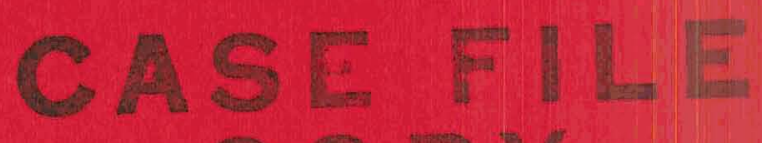

June, 1971

TR-E 39

COPY

\section{DEPARTMENT OF ENERGY ENGINEERING-}


A TURBULENCE DESCRIPTION OF COUETTE ELOW

\author{
by \\ Paul M. Chung \\ Department of Energy Engineering \\ University of Illinois at Chicago
}

*This work is supported by the National Aeronautics and Space Administration. NGR 14-012-012. 
ABSTRACT

The simplified statistical theory developed earliex by the present author is made self-containing for the wall shear filows. Duxing the course of the formulation, the comparison of the present theory, developed srom the generalized Browian motion, with the stochastic theory based on the Navier-stokes equation, was made. The mutual consistency of the two theories was shown. Analysis of the resulting equations shows that the flow field naturally divides itself into the three layers of the outer, transition, and the inner layers. The contribution of the small edaies through viscosity to all observable propexties is negingible in the outer layer, whereas its contribution to the mean shear is of tixst order in the transition layer. In the inner layer, all viscosity effects are of first order. The comparison of the couette flow solutions obtained with the availabie experimental results shows that the present theory describes the rather detailed turbulence structure of the flow field satisfactorily in addition to correctly predicting the mean velocity profile and the surface shear. 


\section{INTRODUCTION}

Most of the real turbulent shear flows defy analysis by the classical stastical turbulence theories because of the extreme complexity of the theories. Studies of these flows, thexefore, have exclusively depended on the phenomenological theories such as the Prandtl's mixing-length theory. (See for instance, References 1 - 6.) Many of the important aspects of the flow, howevex, such as the turbulence structure of the flow field and the chenical reaction, cannot be described correctly by these phenomenological theories,

A simplified statistical theory was developed by the present author ${ }^{(7-9)}$ which contained those stochastic properties of the high Reynolds number turbulence field which are critical in determining the salient turbulence and transport characteristics of the shear flow fields. It has been demonstrated $(8,9)$ that this theory can indeed be employed to describe tractably the various prom perties of a shear flow field, such as the turbulerice and chemical structures of a turbulent diffusion flame; which has not been possible before.

The previous applications ${ }^{(7-9)}$ of the simplified statistical theory were Iinited to the shear fiow fielas with no solid boundaries. The theory was first applied ${ }^{(7)}$ to the couette flow at one Reynolas number as the initial test of the theory. However, the expeximentally determined dissipation function of a pipe flow available at that Reynolds number had to be employed: Also, sevexal assumptions had to be made in order to apply the theory all the way to the wall.

In this paper, the structure of a flow field as affected by a solid boundary will be described by the simplified statistical theory with the same rigor as that previously applied to the flow fields $(8,9)$ with no solid boundaries. For this purpose, the plane couette flow is chosen because of its simple flow configuration. The fluid is considered to be incompressible and chemically inert. 
It will be shown that, when the Reynolds number is sufficiently large, the flow field naturally divides itself into three layers: the outex layer, intermediate (transition) layer, and the inner layer. Solution of the governing equations leading to such division will be carried out by the method of matched asymptotic expansions.

The subdivision of the flow field into such three layers, and even the description of the field by the matched asymptotic expansions, are not new in themselves. (See for instance, Reference 10.) In the previous works, however, these subdivisions were the results of the analysis of the phenomenologically constructed equations with the emperical functions and parameters a priori. supplied from the known experimental results. Therefore, the analysis essentially returned what one put in a priori emperically. Furthermore, the division and the asymptotic matching were all based on the mean velocity profile only.

In contrast, the present theory is self-contained. Hence, it is the natural scaling of the governing equations, that control the behavior of the Reynolds stress, turbulence energy, etc., as well as the mean velocity, which results in the division into such layers.

From the present analysis, the mean velocity, turbulence energy, and the Reynolds stress profiles as well as the surface shear stress will be determined as functions of the flow Reynolds number. These results will be compared with the available experimental data of Reichardt ${ }^{(4)}$ and Robertson, et.al. $(5,6)$ A detailed description of the turbulent Couette flow, and that of the general near-surface turbulence structure of shear flows, will be given in terms of the present theory. 


\section{GOVERNING FOKKER-PIANCK EQUATION}

The detailed development of the present simplified statistical theory is given in the previous papers, ${ }^{(7-9)}$ and it will not be repeated here. It is only pointed out here that the theory resulted in a set of modified Fokkerm Planck equations governing the distribution functions of the fluid elements and the chemical species. These equations have been made self-containing $(8,9)$ For the flow fields with no solid boundaries.

In the present section, the Fokker-Planck equation governing the distribution function of the fluid elements will be made self-containing for the shear flows with solid boundaries.

In the next section, a set of moment equations will be derived from the Fokker-Planck equation in order. to solve this equation by the moment method due to Liu and Lees ${ }^{(11)}$ as it was done in the earlier problems. ${ }^{(7-9)}$ The solution of the moment equations for Couette flow and the discussion of the results will then follow.

\section{General Form}

The Fokker-planck equation which governs the distribution function of the fluid elements, $f(t, \vec{x}, \vec{u})$, was formulated $(7,8)$ as,

$$
\frac{\partial f}{\partial t}+u_{j} \frac{\partial f}{\partial x_{j}}+\frac{\partial}{\partial u_{j}}\left(f k_{j}\right)=\beta\left\{\frac{\partial}{\partial u_{j}}\left[f\left(u_{j}-u_{o j}\right)\right]+\frac{\left\langle U_{k} U_{k}\right.}{3} \frac{\partial^{2} E}{\partial u_{j} \partial u_{j}}\right\}
$$

The distribution function is defined such that $f(t, \vec{x}, \vec{u})$ du denotes the probability of finaing a fluid element in the phase cell du about the instantaneous absolute 
velocity $\vec{u}$, at time $t$ and position $\vec{x}$. The cartesian tensor notation is employed in Eq. (1). The symbol $u_{0 j}$ represents the average velocity whereas $U_{j}$ is the instantaneous velocity relative to $u_{0 j}$. Hence, $v_{j}=u_{j}-u_{0 j}$. The symbol<> denotes the ensemble average. Thus, for instance,

$$
\begin{aligned}
& \left\langle U_{K} U_{K}\right\rangle=\int f U_{K} U_{K} d \vec{u} \\
& \left.<u_{j}\right\rangle=u_{o j}=\int f u_{j} d \vec{u}
\end{aligned}
$$

The quantity $f$ represents the characteristic relaxation rate of the nonequilibxium degree of freedom representing the larger energy-containing eddies. $K_{j}$ represents the effects on the fluid elements of the molecular viscosity and the applied mean pressure gradient.* These two quantities, $\beta$ and $\mathrm{K}_{\mathrm{j}}$, must be properly defined in order to rendex $\mathrm{Eq}$. (1) self-containing.

The characteristic relaxation rate, 8 , is given $(7,8)$ as,

$$
\beta=\frac{\left\langle U_{K} U_{k}>^{1 / 2}\right.}{2 \lambda}\left(I+\frac{I_{1}}{U_{O^{\infty}}} \frac{\partial u_{0}}{\partial y}\right)
$$

for the two-dinensional flows wherein the predominant mean velocity gradient is $\partial u_{0} / \partial y$. The quantities $u_{0}$ and $y$ are the mean velocity in the streamwise direction and the ordinate normal to the streamwise direction, respectively. Is and $\lambda$ are the characteristic lengths respectively of the flow field and the system producing the edaies. In a pure shear-produced turbulence, $\lambda=L$ when $L$ is taken as the shear layer thickness. The subscript $\infty$ denotes the reference condition. 
The expression for $\mathrm{k}_{j}$ will now be discussed for the flows wherein the mean pressure gradient is negligible.

\section{Wa11 Effect}

The present turbulence model ${ }^{(7-9)}$ is built upon the known phenomenon $(12,13)$ of the statistical separation which exists between the nonequilibrium, energycontaining, larger eddies and the smaller, equilibrium eddies when the turbulence Reynolds number is sufficiently large. All observable properties of a turbulence field and also the dissipation rate are determined by the behavior of these larger eddies. The lower wave number region of the spectrum signifying the energy-containing eddies is represented by one nonequilibrium degree of freedom whose characteristic relaxation rate is given by Eq. (3).

When there is no solid boundary, this nonequilibrium degree of freedom is sufficient to determine both the observable properties and the dissipation rate. Hence, it was formulated ${ }^{(8)}$ for the free turbulence

$$
K_{j}=-\beta^{v}\left(u_{j}-u_{o j}\right)
$$

with

$$
B^{v}=B
$$

where $\beta^{\nu}$ is the characteristic dissipation rate.

As a solid boundary is approached in a flow, the characteristic length of the nonequilibrium degree of freedom is expected to decrease, and this decrease is manifested by the usual increasing of the mean velocity gradient near the wall. 
This effect of the decreasing characteristic length on the chaxactexistic rem laxation rate of the nonequilibrium degree of freedom is already included in Eqs. (3) through the mean velocity gradient.

There is, however, another influence of the solid boundary. As the solid boundary is approached, the energy-containing region of the spectrum broadens; thus, the inertial subrange moves toward the higher wave nurber region. rhis is clearly seen, for instance, in the figures 4.4 and 4.5 of Hinze (13) which show the energy spectra of the pipe and boundary layer flows obtained by Jaufer ${ }^{(14)}$ and Klebanoffy, et.al. (15)

As it was mentioned earlier, the dissipation rate is controlled by the energy-containing eddies when the turbulence Reynolds number is sufficienty large. More strictly speaking, however, it is controlled by the region adjacent to the low wave number end of the inertial subrange $(12,13)$ which corresponds to the high wave-number end of the energy containing region. This upper end of the energy-containing region moves into the higher wave number much faster then does the ronequilibrium degree of freedom representing the energy-containing region as a whole, as the wall is approached. That is, the eddies controlIing the dissipation rate are nore directly affected by the solid boundary as compared to the energy-containing eadies as a whole. $\mathrm{K}_{\mathrm{j}}$, therefore, must reflect this direct effect of the solid boundary on the dissipation with the approaching of the boundany.

Also, the increasing role of the small, equilibrium (near-equilibrium) ediles on the observable properties must be manifested in $k_{j}$. since these edaies are smali in size and in near-equilibrium, their contributions to the observable properties should be in local in character. This means that their contributions should be functions of the local properties, and not functions of the two-point correlations, etc., which are the properties of the larger eddies 
expressed in the present theory by the characteristic relaxation rate $\mathrm{f}$. In view of the above biscussion, the $K_{j}$ is expressed for the wall-turbuIence as,

$$
k_{j}=-8^{\nu}\left(u_{j}-u_{0 j}\right)-k_{j}^{\nu}
$$

with, for the conette flow,

$$
\beta^{\nu}=\frac{\left\langle U_{K} K>^{1 / 2}\right.}{2 \lambda}\left(I+\frac{I}{U_{0}} \frac{\partial u_{0}}{\partial y}\right)_{\infty} \frac{I}{Y+Y^{*}}
$$

In eq. (6), the first term represents the effect of molecular viscosity on the dissipation whereas the second term, $\mathrm{K}_{j}^{\nu}$, that on the observable properties.

In Eq. (7) defining the characteristic dissipation rate, the characteristic size of the eddies responsible for the energy input to the inertial subrange is considered to be of the oxder of the distance from the wall. However, as $y \div 0$, the size of these eddies should not become zero on physical grounds. In the flow region near a wall, the minimum size, $y^{*}$, of the edaies which can be considered as being at the low wave number end of an inertial subrange must be of the order of the thickness of the layer within which the viscosity effect on the observable properties is of first order.

As it will be seen in a subsequent section, the viscosity effect first manifests itself on the mean shear as the wall is approached. This Iayer will be called the "transition layer". The minimu size of the eddies, $y^{*}$, is. therefore, of the order of the transition layer. This quantity will be defined more precisely in section IV.

It is clear that the transition layer thickness and, therefore, $y^{*}$ will be functions of the flow Reynolds number. Furthermore, $y * / L<1$ for the large flow 
Reynolas numers of the present interest. Therefore, $B$ defined by Eq. ( becomes equal to the $\beta$ defined by Eq. (3) as $y \rightarrow L$. That is, in the outemoet region of a turbulent shear layex where the influence of the wall is megligible, the dissipation rate is controlled by the same nonequilibrium degree of freedom which controls the observable properties, consistent with the previous analysis $(8,9)$ of the free turbulence.

$k_{j}^{\nu}$ will now be considered.

Viscosity Effect on Observable Properties, and Comparison with the Lundgren"s and Fox $\mathrm{s}^{(17)}$ Theories

As it was mentioned earliex, the influence of the smaller edaies on the distribution function, $f$, cannot be neglected near the wall.

The Reynolds number based on the mean fluctuation velocity and the scale of these edies is very small. Hence, the statistical behavior of these edies and their effect on E cannot be determined by the present generalized Brownin stochastios. $(7,8)$

Also, the small Reynolds number of these eddies means that their contribum tion to the distribution function and to the observable properties is dependent upon the molecular viscosity.

The effect of these small edaies on $f$, that is, the expression for $k^{V}$ will be obtained from the Navier-stokes equation following the stochastic analyses of that equation given by Iundgren (16) and Fox. (17) By comparing the present Fokker-planck equation to the equations derived from the Navier-stokes equation. the coneistency of the present theory with that developed by Lundgren (16) and Eox (17) can also be seen. 
In the works of Lundgren and Fox, it is first considered that there exists an n-point distribution function of fluid elements, $f(n)\left(t, \vec{x}_{1}, \vec{x}_{2}, \cdots,-\cdots, \vec{x}_{n}, \vec{v}_{2}, \vec{v}_{2}\right.$ ? $-\left(-\vec{v}_{n}\right)$, where $n$ may be as large as it is necessary to describe a turbulence field. Then the detailed ensemble average of any function $G\left[\vec{v}_{1}\left(\vec{x}_{1} t\right), \vec{v}_{2}\left(\vec{x}_{2}, t\right), \cdots\right.$ $\left.--\vec{v}_{n}\left(\vec{x}_{n}, t\right)\right]$ is given by

$$
\bar{G}=\int E^{(n)} G d \vec{v}_{1} d \vec{v}_{2} \cdots \cdots \cdot \cdots \vec{v}_{n}
$$

The bar on a function is employed to represent the particular ensemble averaging defined by Eq. (8).

The Iower hierarchy distribution function is then defined in terms of $f^{(n)}$ and the Dirac delta function as,

$$
\begin{aligned}
& f^{(1)}\left(t, \vec{x}_{I}, \vec{u}_{1}\right)=f\left(t, \vec{x}_{1}, \vec{u}_{I}\right)=\frac{\delta\left[\vec{v}_{I}\left(\vec{x}_{1}, t\right)-\vec{u}_{1}\right]}{}, \\
& f^{(2)}\left(t, \vec{x}_{1}, \vec{x}_{2}, \vec{u}_{1}, \vec{u}_{2}\right)=\frac{\delta\left[\vec{v}_{1}\left(\vec{x}_{1}, t\right)-\vec{u}_{1}\right] \delta\left[\vec{v}_{2}\left(\vec{x}_{2}, t\right)-\vec{u}_{2}\right]}{}
\end{aligned}
$$

ete.

The one-point aistribution $f\left(t, \vec{x}_{2}, \vec{u}_{y}\right)$ defined above is the same as the present distribution function $E(t, \vec{x}, \vec{w})$.

From Eq. $(\theta)$,

$$
\frac{\partial \varepsilon}{\partial t}=-\frac{\partial \vec{v}_{I}}{\partial t} \cdot \frac{\partial}{\partial \vec{u}_{I}} \hat{\delta}\left(\vec{v}_{1}-\vec{u}_{1}\right)
$$

Now, by obtaining the expression for $\frac{\partial \vec{v}}{\partial t}$ in terms of space derivatives from the Navier-Stokes equation, and by substituting this expression into Eq. (10), Lundgren (16) dexived the following equation for $E$ : 


$$
\begin{aligned}
& \frac{\partial E}{\partial t}+u_{j} \frac{\partial f}{\partial x_{j}}+\frac{\partial}{\partial u_{j}}\left\{-\frac{1}{4 \pi} \int\left(\frac{\partial}{\partial x_{k}} \frac{1}{\left|\vec{x}-\vec{x}_{2}\right|}\right)\left(u_{2 m} \frac{\partial}{\partial x_{2 m}}\right)^{2} f_{2 k} z_{2 k} \partial u_{2 j}\right\}
\end{aligned}
$$

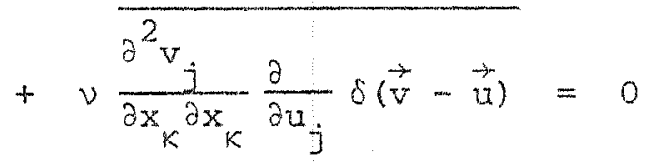

where $v$ is the kinematic viscosity.

In the present theory, the distribution function when the mean pressure gradient is negligible is governed by the Fokker-Planck equation, Eq. (I), which becomes with $k_{j}$ defined by $E q .(6)$,

$$
\begin{gathered}
\frac{\partial f}{\partial t}+u_{j} \frac{\partial f}{\partial x_{j}}-\beta\left\{\frac{\partial}{\partial u_{j}}\left[f\left(u_{j}-u_{0 j}\right)\right]+\frac{<U_{K} U^{>}}{3} \frac{\partial^{2} E}{\partial u_{j} \partial u_{j}}\right\} \\
-\beta^{v} \frac{\partial}{\partial u_{j}}\left[f\left(u_{j}-u_{o j}\right)\right]-\frac{\partial}{\partial u_{j}}\left(f K_{j}^{v}\right)=0
\end{gathered}
$$

A comparison of the Iundgren's equation, Eq. (II), with the present equation, Eq. (12), shows that the evolution toward the isotropy* is governed by the third term of each equation.

The influence of the molecular viscosity on $f$ is given by the last term of Eq. (11) which must be equal to the last two terms of Eq. (12). In the initial development of the present theory given in reference 7 , the entire viscosity effect, $\mathrm{K}_{j}$, was expressed as,

$$
k_{j}=v \frac{\partial^{2} u_{j}}{\partial x_{k} \partial x_{k}}
$$

When Eq. (13) is employed, the last two terms of Eq. (12) simply becomes,

* Relaxation of the nonequilibrium degree of freedom toward equilibrium. 


$$
\frac{\partial}{\partial u_{j}}\left(f k_{j}\right)=v \frac{\partial}{\partial u_{j}}\left(\sum \frac{\partial^{2} u_{j}}{\partial x_{k} \partial x_{k}}\right)
$$

The Iast term of Eq. (II) can be also written, with the use of Eqs. (8) and (9), as,

$$
v \frac{\partial^{2} v_{j}}{\partial x_{k} \partial x_{k}} \frac{\partial}{\partial u_{j}} \delta(\vec{v}-\vec{u})=v \frac{\partial}{\partial u_{j}}\left(E \frac{\partial^{2} u_{j}}{\partial x_{k} \partial x_{k}}\right)
$$

Therefore, the effect of viscosity on $f$ expressed by Lundgren (16) is identical to that initialy developed in the present theory. (7)

The expression, $f\left(\partial^{2} u_{j} / \partial x_{k} \partial x_{k}\right)$, however, depends on the two-point distribution function $E^{(2)}$ as well as on $E$, as was shown by Lundgren (16) and Fox. (17) Hs it was discussed earlier, the dissipation rate in the high Reynolds-rumber turbulence is controlied by the larger energy-containing eddes. Hence, thet portion of $f\left(\partial^{2} u_{j} / \partial x_{K} \partial x_{k}\right)$ pertaining to dissipation must depend on the twopoint distribution function. On the other hand, as it was explained in the paragraphs preceaing Eq. (6), the contribution of the smali, near-equilibrium eddies on the observable properties, such as $f$, must be local in character. Thus, it must depend onjy on the one-point distribution function $E$.

In the work of reference 7 , the dissipation portion of $f\left(\partial^{2} u_{j} / \partial x_{k} \partial x_{k}\right)$ which depends on the twompoint distribution function was supplied from the available experimental data. In the subsequent analyses of references 8 and 9 , the dissipation was expressed in terms of the evolution rate of the larger eddies as Eqs. (4) and (5). The contribution of the small eddies on through viscosity is negligible when there is no solid boundary. Therefore, the Fokjex-mlanck equation was made self-containing with Eqs. (3) through (5) for free turbuience. That portion of $f\left(\partial^{2} u_{j} / \partial x_{k} \partial x_{k}\right)$ pertaining to the contribution of the small eddes to $f$ is now determined by equating the last two terms of Eq. (12) to the 
last term of Eq. (II). Before these terms are equated, however, the last term of Eg. (1.1) is manipulated into a more convenient form as follows.

By expressing the Dirac delta function as the limit of a Gaussian function, Pox (17) obtained,

$$
\begin{aligned}
& \overline{v \frac{\partial^{2} v_{j}}{\partial x_{k} \partial x_{k}} \frac{\partial}{\partial u_{j}} \delta(\vec{v}-\vec{u})}=\lim _{\zeta \rightarrow 0} \frac{2 v}{\pi^{3 / 2} \zeta}\left[-\frac{\zeta^{2}}{2} \frac{\partial^{2}}{\partial x_{k} \partial x_{K}}-\frac{\partial v_{m}}{\partial x_{k}} \frac{\partial v_{m}}{\partial x_{K}}\right. \\
& \left.+\frac{2\left(v_{m}-u_{m}\right)\left(v_{n}-u_{n}\right)}{\zeta^{2}} \frac{\partial v_{m}}{\partial x_{k}} \frac{\partial v_{n}}{\partial x_{k}}\right] \exp \left[-\left(v_{k}-u_{k}\right)^{2} / \zeta^{2}\right]
\end{aligned}
$$

The above expression is manipulated into a form somewhat different from that obtained by Fox as,

$$
\begin{aligned}
& \overline{\frac{\partial^{2} v_{j}}{\partial x_{K} \partial x_{K}} \frac{\partial}{\partial u_{j}} \delta(\vec{v}-\vec{u})}=-v\left(\frac{\partial^{2} E}{\partial x_{K} \partial x_{K}}-\frac{\partial u_{o m}}{\partial x_{K}} \frac{\partial u_{o n}}{\partial x_{K}} \frac{\partial^{2} f}{\partial u_{n} \partial u_{n}}\right)
\end{aligned}
$$

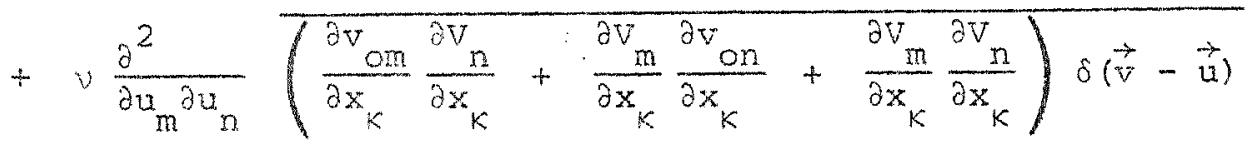

Equation (17) brings out clearly the two aspects of the viscous effect. The first quantity on the right-hand side of $\mathrm{Eq}$. (17) depends on the one-point distribution function only, and it is the contribution of the small edoies to the observable properties. The second quantity, on the other hand, depends on the two-point distribution function, and it is the dissipation term which depends on the larger eddies.

Now, equating the last two terms of $\mathrm{Eq}$. (12) to the right-hand side of Eq. (17), it is seen that the first of the last two terms of Eq. (12) is equal to the last term of $\mathrm{Eq}$. (17), and, 


$$
\frac{\partial}{\partial u_{j}}\left(E k_{j}^{\nu}\right)=v\left(\frac{\partial^{2}}{\partial x_{k} \partial x_{k}}-\frac{\partial u_{o m}}{\partial x_{k}} \frac{\partial u_{o n}}{\partial x_{k}} \frac{\partial^{2} f}{\partial u_{m} \partial u_{n}}\right)
$$

\section{Self-Contained Fokker-Planck Equation}

Equation (12) governing $f$ is now self-contained with the use of $\mathrm{Eq}$. (18). This equation is transformed into the following by considering $f$ to be a function of the relative velocity $\vec{u}$ instead of the absolute velocity $\vec{u}$.

$$
\begin{aligned}
& \frac{\partial f}{\partial t}-\frac{\partial u_{o k}}{\partial t} \frac{\partial f}{\partial U_{k}}+\left(U_{k}+u_{o k}\right) \frac{\partial f}{\partial x_{k}}-\left(U_{k}+u_{o K}\right) \frac{\partial f}{\partial U_{m}} \frac{\partial u_{m}}{\partial x_{k}} \\
& -\beta\left[\frac{\partial}{\partial U_{K}}\left(f U_{K}\right)+\frac{<U_{K} U^{>}}{3} \frac{\partial^{2} E}{\partial U_{m} \partial U_{m}}\right]-\beta^{\nu} \frac{\partial}{\partial U_{K}}\left(f U_{K}\right) \\
& -v\left(\frac{\partial^{2} f}{\partial x_{K} \partial x_{k}}-2 \frac{\partial u_{o k}}{\partial x_{m}} \frac{\partial^{2} f}{\partial x_{m} \partial u_{K}}-\frac{\partial^{2} u_{o k}}{\partial x_{m} \partial x_{m}} \frac{\partial f}{\partial u_{k}}\right)=0
\end{aligned}
$$

The quantities $\beta$ and $\beta^{v}$ are given by Eqs. (3) and (7), respectively. Solution of Eq. (19) for Couette flow and discussion of the results constitute the remainder of this paper. 
III. GOVERNING MOMENT EQUATIOUS

The solution of Eq. (19) will be obtained by the moment method due to Liu and Lees (II) as in the previous papers. (7-9) For this purpose, we fixst obtain the genexalized moment equation for steady state by multiplying Es (19) by a function $Q(\vec{U})$, and integrating it with respect to the velocity space.

$$
\begin{aligned}
& \frac{\partial}{\partial x_{K}} \int E\left(U_{K}+u_{o K}\right) Q \overrightarrow{Q U}+\frac{\partial u_{o m}}{\partial x_{K}} \int E \frac{\partial Q}{\partial U}\left(U_{K}+u_{O K}\right) d \vec{U} \\
& +\beta\left[\int f_{K} \frac{\partial Q}{\partial U_{K}} d \vec{U}+\frac{\left\langle U_{K} U^{\prime}\right.}{3} \int \frac{\partial f}{\partial U_{m}} \frac{\partial Q}{\partial U} d \vec{U}\right]
\end{aligned}
$$

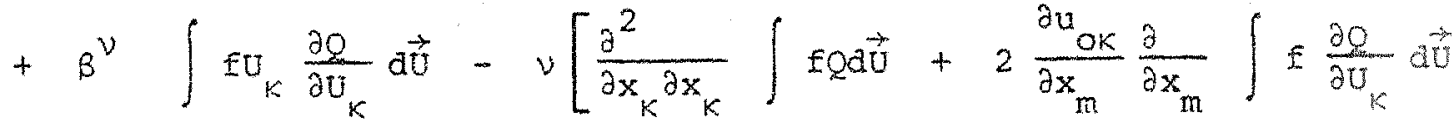

$$
\begin{aligned}
& \left.+\frac{\partial^{2} u_{o k}}{\partial x_{m} \partial x_{m}} \int f \frac{\partial g}{\partial u} \vec{u}\right]=0
\end{aligned}
$$

The particular moment equations governing the couette flow is now dexived from Eq. (20) by first expressing

$$
f=f_{1}+f_{2}
$$

where

$$
\begin{aligned}
& f_{1}=\frac{1}{\left(2 / 3 \pi E_{1}\right)^{3 / 2}}\left[-\frac{\left(u-u_{o 1}\right)^{2}+v^{2}+w^{2}}{2 E_{1} / 3}\right] \text { for } v \geq 0 \\
& f_{2}=\frac{1}{\left(2 / 3 \pi E_{2}\right)^{3 / 2}}\left[-\frac{\left(u-u_{o 2}\right)^{2}+v^{2}+w^{2}}{2 E_{2} / 3}\right] \text { for } v \leq 0
\end{aligned}
$$


and $E_{I}$ and $f_{2}$ ase zero for $V<0$ and $V>0$, respectively*

The coordinate system for the couette flow is given in Fig. 1. Note that

u, $v$, and without the tensor indices are the $x, y$, and $z$ components of the instantaneous absolute velocity, respectively. Also, U, $V$, and without the tensor indices are the $x, y$, and $z$ components of the relative velocity, respectively.

Thexe are four unknown functions of $y$ in the expression for $f$, which are $E_{1}, E_{2}, u_{o 1}$, and $u_{02}$. Four particular moment equations are, therefore, generated Fron Eq. (20) by substituting four different, suitable functions for $q$ in that equation.

In the previous analyses, $(7-9)$ the values chosen for $Q$ were $I, U, U V$, and $U_{K} U_{K}$. In the following analysis, the four values of $Q=1, U, U^{2}$, and $v^{2}$ will be employed, because the resulting moment equations with these values of $Q$ are a Ittle simpler than those resulting from the previous values of $Q$. BY comparing the limiting homologous solution of the present moment equations with the homologous solution obtained earlier, ${ }^{(8)}$ it will be shown that these two sets of $Q$ give about the same results.

First, the substitutions of $\mathrm{Eq} .(21)$ and $Q=1$ into $\mathrm{Eq}$. (20) simply gives,

$$
E_{1}=E_{2}=E
$$

The successive substitution of $Q=U, U^{2}$, and $V^{2}$ along with the substitution of Eq. (21) for $\mathrm{f}$ into Eq. (20) then give a set of three differential equations after some manipulation. These equations are suitably nondimensionalized and written as, 


$$
\begin{aligned}
& \frac{d \psi \theta}{d Y}=\frac{(6 \pi)^{1 / 2}}{2} \varepsilon \frac{d^{2} \phi}{d Y^{2}} \\
& \psi \theta \frac{d \phi}{d Y}+\frac{1}{2}\left(\frac{\pi}{6}\right)^{1 / 2} a\left(\theta_{\infty}^{2}+\frac{\psi_{\infty}^{2}}{4}\right)^{1 / 2}\left(2+\frac{d \phi}{d Y}\right)_{\infty}\left[\frac{2+\frac{d \phi}{d Y}}{\left(2+\frac{d \phi}{d Y}\right)} \psi_{\infty}^{2}\right. \\
& \left.+\frac{2 \theta^{2}+3 / 2 \psi^{2}}{\mathrm{x}+\mathrm{Y}^{*}}\right]-(6 \pi)^{1 / 2} \operatorname{co}^{2} \frac{\mathrm{a}^{2}}{d \mathrm{y}^{2}}\left(\frac{\theta^{2}}{3}+\frac{\psi^{2}}{4}\right)=0 \\
& \frac{1}{2} \alpha\left(\theta_{\infty}^{2}+\frac{\psi_{\infty}^{2}}{4}\right)^{1 / 2}\left(2+\frac{d \phi}{d Y}\right)_{\infty}\left[\frac{\theta^{2}}{Y+Y^{*}}-\frac{2+\frac{d \phi}{d Y}}{\left(2+\frac{\partial \phi}{d Y_{*}}\right)_{\infty}} \frac{\psi^{2}}{4}\right] \\
& -\varepsilon \alpha^{2} \frac{a^{2} \theta^{2}}{d y^{2}}=0
\end{aligned}
$$

where,

$$
\begin{aligned}
& \phi=\frac{u_{01}+u_{02}}{u_{0 \infty}}=2 \frac{u_{0}}{u_{0 \infty}}, \quad \psi=\frac{u_{01}-u_{02}}{u^{*}} \\
& \theta=E^{I / 2} / u^{*}, \quad \alpha=u^{* / u_{O \infty}}, \quad \varepsilon=\frac{1}{\alpha^{2} R e}, \\
& R=\frac{u_{0 \infty} L}{v}, \quad Y=\frac{Y}{L}
\end{aligned}
$$

and $u^{*}$ is the friction velocity defined as $\left(\tau_{w} / \rho\right)^{1 / 2}$ where $\tau_{w}$ is the surface shear stress.

In deriving Eqs. (23) through (25), $\beta$ and $\beta^{\nu}$ given by Eqs. (3) and (7), respectively, have been simplified a little by replacing $\left\langle\mathrm{U}_{\mathrm{K}} \mathrm{U}_{\mathrm{K}}>^{1 / 2}\right.$ by $<\mathrm{U}_{\mathrm{K}} \mathrm{U}_{\mathrm{K}}>^{1 / 2}$ The only undefined parameter in Eqs. (23) through (25) is $\mathrm{Y}^{*}=y^{*} / \mathrm{I}$ where $y^{*}$ is the minimum eday scale explained in the sentences following Eq. (7). As 
it was explained there, $y^{*}$ is of the order of the txansition layer thickness. It will, therefore, be determined in the next section during the discussion of the natural scaling of the various flow regions.

Finally, the boundary conditions are given at the two solid boundaries as follows:

At $X=0$

$$
\phi=\psi^{2}=\theta^{2}=0
$$

At $Y=2$

$$
\begin{aligned}
& \phi=4 \\
& \psi^{2}=\theta^{2}=0
\end{aligned}
$$

In the subsequent solution, however, Eqs. (28) will be replaced by a more convenient set of boundary conditions at $\mathrm{Y}=1$. 
IV. BEHAVTOR OF THE GOVERNING MOMENT EQUATIONS

A study of Eqs. (23) through (25) shows that there are two small paxameters, $\varepsilon$ and $\alpha$, which govern the general behavior of these equations when Re js large.

It will be shown in this section that the scaling based on these two parameters naturally divides the flow field into the three regions of outer, transition, and inner layers. The solution of Eqs. (23) through (25) will then be obtained in the next section by the method of matched asymptotic expansions for the large Reynolds numbers.

We consider that the Reynolds number is sufficiently large such that there exists the following order relationship.

$$
\varepsilon \ll \alpha<1
$$

Each of the three layers will be discussed in the following for $0 \leq Y \leq 1$.

\section{Outer Layer}

This layer is defined as that region wherein " $Y=O(1)$. We expand the dependent variables as,

$$
\begin{aligned}
& \phi(\varepsilon, O, Y)=\phi_{O O}(Y)+\varepsilon \phi_{10}(Y)+\alpha^{2} \phi_{O I}(Y)+o\left(\varepsilon^{2}, \alpha^{4}, \varepsilon \alpha^{2}\right) \\
& \psi^{2}(\varepsilon, \alpha, Y)=\psi_{O O}^{2}(Y)+\varepsilon \psi_{10}^{2}(Y)+\alpha^{2} \psi_{O 1}^{2}(Y)+0\left(\varepsilon, \alpha_{1}^{4} \varepsilon \alpha \alpha^{2}\right) \\
& \theta^{2}(\varepsilon, \alpha, Y)=\theta_{O O}^{2}(Y)+\varepsilon \theta_{10}^{2}(Y)+\alpha^{2} \theta_{O I}^{2}(Y)+0\left(\varepsilon^{2}, \alpha^{4}, \varepsilon \alpha^{2}\right)
\end{aligned}
$$


A subtitution of the above series into Egs. (23) through (25) gives. to first order,

$$
\frac{d \omega^{\theta} \infty}{d x}=0
$$

$$
\begin{aligned}
& \psi_{000}^{\theta} \frac{\alpha \phi}{d x}+\frac{1}{2}\left(\frac{\pi}{6}\right)^{1 / 2} \alpha\left(\theta_{\infty}^{2}+\frac{\psi_{\infty}^{2}}{4}\right)^{1 / 2}\left(2+\frac{d \phi}{d x}\right) \\
& {\left[\frac{2+\frac{\partial \phi}{\partial q}}{\left(2+\frac{\partial \phi}{d q}\right)} \psi_{\infty}^{2}+\frac{2 \theta_{00}^{2}+3 / 2 \omega_{\infty}^{2}}{\Psi}\right]=0}
\end{aligned}
$$

$$
\frac{\theta^{2}}{Y}-\frac{2+\frac{\partial \phi}{\partial Y}}{\left(2+\frac{d \phi}{d X}\right)_{\infty}} \frac{\psi^{2}}{4}=0
$$

In view of the order relationships set forth by Eqs (29), the terms of $O(\alpha)$ are consistenty retaned along with the terms of $O(1)$ * Also, as we

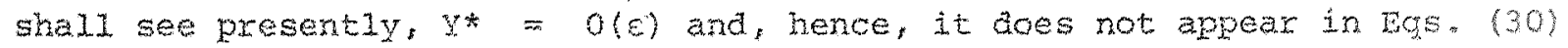
through (32).

Equations (30) through (32) show that, to the first order, the effect of the smaliex edaies through viscosity on the observable properties is regligible in this layer. This is evident from the fact that the second order derivative terms axe completely absent in these equations.

As it was explained in the paragraphs following Eg. (7), that equation is constructed with the condition that the effect of the wall on the aissipation is negligible at $Y=1$. This means that the present

* Such is the case with the other two layers also. 
equations should describe at $\mathrm{Y}=I$ a homologous flow analyzed earlier. (8)

fact that the flow is homologous at the center of a couette flow when Re is

large has been experimentaliy established by Robertson.

Equation (30) through (32) can be readily solved, and there resuIt, at $Y=1$,

$$
\psi_{\infty}=-\frac{2}{(3 \pi)^{1 / 2}} \frac{1}{\alpha}\left(\frac{d \phi_{o O} / d Y}{2+d \phi_{O O} / d Y}\right), \quad \theta_{\infty O}=\frac{1}{(3 \pi)^{1 / 2}} \frac{1}{\alpha}\left(\frac{d \phi_{o O} / d Y}{2+d \phi_{o O} / d Y}\right)
$$

where

$$
\alpha=\left(\frac{2}{3 \pi(6 \pi)^{I / 2}}\right)^{I / 2}\left(\frac{d \phi_{o O} / d Y}{2+d \phi_{O O^{\prime}} d \bar{X}}\right)
$$

The Reynolds stress and the turbulence energy can be constructed from $\psi_{0}$ and $\theta_{00}$ as (see Ref. 8),

$$
\begin{aligned}
& \frac{\Delta U V\rangle}{u_{\infty}^{2}}=\frac{\alpha^{2}}{(6 \pi)^{1 / 2}} \psi_{\infty 0^{\theta}}{ }^{\theta} \\
& \frac{\left\langle U_{K} U_{K}\right\rangle}{2}=\alpha^{2}\left(\theta_{\infty}^{2}+\frac{\psi_{\infty O}^{2}}{4}\right)
\end{aligned}
$$

As it was explained following Eqs. (22), the present governing equations, Eqs. (23) through (25), are obtained by taking the different moments of the Fokker-Planck equation rather than the moments taken in reference 8 . A comparison of Eqs. (33) and (34) with the corresponding solutions of reference 8 shows that the two sets of solutions agree within $10 \%$.

Figure 2 shows the Reynolds stress and turbulence energy computed by Egs. (35) and (36), and those computed in the earlier work. (8) experimental results of Reichardt ${ }^{(4)}$ and Robertson ${ }^{(5)}$ obtained at the center of Couette flow. 


\section{Transition Layer}

This layer is defined as that region of $\mathrm{X}=O(\varepsilon)$ from the wall.

We first define the stretched independent variable as,

$$
n=Y / \varepsilon
$$

The dependent variables are expanded in the manner same as Eqs. (29).

$$
\begin{aligned}
& \phi(\varepsilon, \alpha, \eta)=\hat{\phi}_{o 0}(\eta)+\varepsilon \hat{\phi}_{10}(\eta)+o\left(\alpha^{2}\right) \\
& \psi^{2}(\varepsilon, \alpha, \eta)=\hat{\psi}_{o \infty}^{2}(\eta)+\varepsilon \hat{\psi}_{10}^{2}(\eta)+o\left(\alpha^{2}\right) \\
& \theta^{2}(\varepsilon, \alpha, \eta)=\hat{\theta}_{o 0}^{2}(\eta)+\varepsilon \hat{\theta}_{10}^{2}(\eta)+o\left(\alpha^{2}\right)
\end{aligned}
$$

A substitution of the above series into Eqs. (23) through (25) gives, to the first order,

$$
\begin{aligned}
& \frac{d \hat{\psi} \hat{\theta}_{0 O}}{\mathrm{~d} \eta}=\frac{(6 \pi)^{1 / 2}}{2} \frac{\mathrm{d}^{2 \hat{\phi}} \mathrm{OO}}{\mathrm{d \eta ^{2 }}}
\end{aligned}
$$

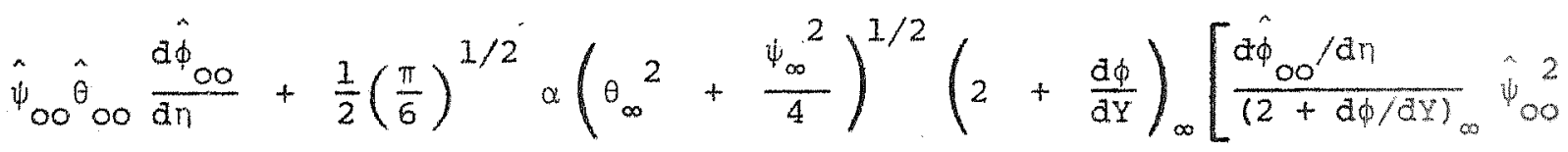

$$
\begin{aligned}
& \left.+\frac{\hat{\theta}_{00}^{2}+\frac{3^{\hat{2}} \hat{\psi}^{2}}{20}}{\left(\eta+n^{*}\right)}\right]=0 \\
& \frac{\hat{\theta}_{o O}^{2}}{n+n^{*}}-\frac{\hat{d \phi}_{O O} / d \eta}{(2+d \phi / d Y)_{\infty}} \frac{\hat{\psi}_{O O}^{2}}{4}=0
\end{aligned}
$$


First we notice in the above equations that the second order derivative term appears in the momentum equation, Eq. (39). This means that the contri-bution of the small eddies through viscosity to the momentum transport is of first order in this layer. However, the contributions to the energies, $\left\langle 0^{2}>\right.$ and $\left\langle v^{2}\right\rangle$, are still negligible.

The above points were first mentioned in the paragraphs following Eq. (7). It was also explained there that $y^{*}$ should be of the order of the transitionlayer thickness, that is $Y^{*}=O(\varepsilon)$. The present solution is not too sensitive to the particular choice of the value for $\mathrm{Y}^{*}$ provided that it is $O(E)$. It is likely that $\mathrm{Y}^{*}<\epsilon$ because the size of the edaies generated by a shear layex is always smaller than the layer thickness.

We let, in the present study,

$$
Y^{*}=E / 3,\left(\eta^{*}=1 / 3\right)
$$

\section{Inner Layer}

This layer is defined as that region adjacent to the wall whose thickness is of the order $\varepsilon \alpha$. Note that $\varepsilon \alpha$ is the reciprocal of $u \star I / v$ which is of the order of the turbulence Reynolds number.

The stretched independent variable is defined as,

$$
\xi=\eta / \alpha=Y / \varepsilon \alpha=\frac{u^{*} y}{v}
$$

Note that the inner variable, $\xi$, is the usual nondimensionalized ordinate with which the various experimental results near the wall are correlated. 
The dependent variables are expanded as,

$$
\begin{aligned}
& \phi(\varepsilon, \alpha, \xi)=\alpha \Phi_{01}(\xi)+\varepsilon \Phi_{10}(\xi)+o\left(\alpha^{3}, \varepsilon^{2} \alpha \varepsilon\right) \\
& \psi^{2}(\varepsilon, \alpha, \xi)=\Psi_{00}^{2}(\xi)+\varepsilon_{10}^{2}(\xi)+0\left(\alpha^{2}, \varepsilon^{2}, \alpha^{2} \varepsilon\right) \\
& \theta^{2}(\varepsilon, \alpha, \xi)=\theta_{00}^{2}(\xi)+\varepsilon_{10}^{2}(\xi)+0\left(\alpha, \varepsilon^{2}, \alpha^{2} \varepsilon\right)
\end{aligned}
$$

Substituting the above series into Eqs. (23) through. (26), we obtain, to first order,

$$
\begin{aligned}
& \frac{d \Psi . \theta 00}{d \xi}=\frac{(6 \pi)^{1 / 2}}{2} \frac{d^{2} \phi 1}{d \xi^{2}}
\end{aligned}
$$

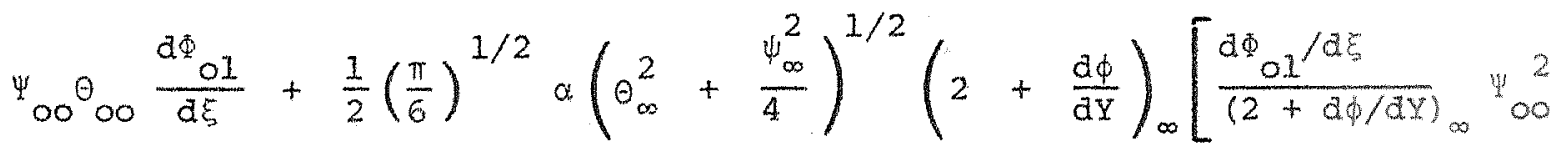

$$
\begin{aligned}
& \left.+\frac{2 \theta_{00}^{2}+\frac{3}{2} \Psi_{00}^{2}}{\eta^{*}}\right]-(6 \pi)^{1 / 2} \frac{\mathrm{a}^{2}}{\mathrm{~d} \xi^{2}}\left(\frac{\theta_{00}^{2}}{3}+\frac{\Psi_{00}^{2}}{4}\right)=0 \\
& \frac{\alpha}{2}\left(\theta_{\infty}^{2}+\frac{\Psi_{\infty}^{2}}{4}\right)^{1 / 2}\left(2+\frac{d \phi}{d Y}\right)_{\infty}\left[\frac{\theta_{\infty}^{2}}{\eta^{*}}-\frac{d \Phi o I / d \xi}{(2+d \phi / d Y)_{\infty}} \frac{\Psi_{0 O}^{2}}{4}\right]-\frac{d^{2} \theta_{\infty}^{2}}{d \xi^{2}}=0
\end{aligned}
$$

We see in the above equations that all the second order derivatives are of first order in this layer. The contributions, therefore, of the smaIl eddies through viscosity to ald observable properties are important in this layer. In fact, it is the viscosity which enables the flow field to finally satisfy the wall boundary conditions given by Eqs. (27) and (28). 
V. SOLUTION FOR HIGH REYNOLDS NUMBERS

The first order equations for the three layers developed in the preceding section can be separately solved and asymptotically matched. However, Eqs. (23) through (25) show that the outer and transition layers can be analyzed sogether with no added mathematical difficulty. By handing these layers together, one can eliminate one matching thus improving the accuracy of the final results.

In this section, the first order equations for the outer-transition layer* and the corresponding equations for the inner layer will be formulated. The solutions of these equations will then be obtained.

Finally, these solutions will be asymptotically matched thus constructing a uniformly valid solution for the entire flow field.

Before we begin the analysis, however, the boundary conditions, Eqs. (28), will be replaced by a set of more convenient ones.

Boundary conditions

The boundary conditions at $Y=0$ are those given by Eqs. (27). Equations (28) will be replaced by a set of the conditions, at $Y=I$ to be determined in the following manner.

As it was mentioned earlier, it has been experimentally established (5) that a homologous condition prevails at the center of a couette flow when Re is sufmficiently large. The homologous solutions have been obtained previously ${ }^{(8)}$ and also in section IV as Eqs. (33) and (34). The boundary conditions at $y=2$ are, therefore, replaced by the homologous conditions at $\mathrm{y}=1$.

This means that we seek the solution of Eqs. (23) through (25) which satisfies the boundary conditions at $\mathrm{Y}=0$ given by Eqs. (27), and the particular

*Combined layer consisting of the outer and transition layers. 
combinations of $\psi_{\infty}^{2}, \theta_{\infty}^{2}$ and $(\mathrm{d} \phi / \mathrm{dY})$ which satisfy the homologous solution. Egs. (33), $a t y=1$. In addition, the solution must satisfy the condition of $(1)=2$. Equations (27) and the conditions at $y=1$ specified above constitute seven boundary contitions whereas gas. (23) through (25) comprise a sixth ordex systen. However, these equations contain an unspecified parameter $\varepsilon$. Therefore, for a perticular set of the boundary conditions given at $Y=1$, there exists a particular value of $\varepsilon$ for which Eqs. (23) through (25) can be solved to satisfy the seven boundary conditions. Since $\varepsilon$ is a unique function of Re, each solution coxresponds to a particular Re.

\section{Outer-rransition Layer}

In order to combine the transition layer with the outer layex, the terms of o(c) must be retained. Thus, we expand,

$$
\begin{aligned}
& \phi(\alpha, Y)=\phi_{0}(Y)+\alpha_{1}^{2} \phi_{1}(Y)+o\left(\alpha^{4}\right) \\
& \psi^{2}(\alpha, Y)=\psi_{0}^{2}(Y)+\alpha^{2} \psi_{1}^{2}(Y)+o\left(\alpha^{4}\right) \\
& \theta^{2}(\alpha, Y)=\theta_{0}^{2}(Y)+\alpha^{2} \theta_{3}^{2}(Y)+o\left(\alpha^{4}\right)
\end{aligned}
$$

As before, the terms of $O(x)$ titil be consistenty xetained for the first order in view of the above expansions.

A substitution of Eqs. (48) into Egs. (23) through (25) gives, to fixst order,

$$
\frac{d \omega_{0} \theta}{d Y}=\frac{(6 \pi)^{1 / 2}}{2}=\frac{a^{2} \phi}{d Y^{2}}
$$




$$
\begin{aligned}
& \psi_{0} \theta_{0} \frac{\partial \phi}{d Y}+\frac{1}{2}\left(\frac{\pi}{6}\right)^{1 / 2} 0\left(\theta_{\infty}^{2}+\frac{\psi_{\infty}^{2}}{4}\right)^{1 / 2}\left(2+\frac{d \phi}{\partial x}\right)_{\infty}\left[\frac{2+d \phi d y}{(2+d \phi / d Y)} \psi_{0}^{2}\right. \\
& \left.+\frac{2 \theta^{2}+\frac{3}{2} \psi_{0}^{2}}{y+(\varepsilon / 3)}\right]=0 \\
& \frac{\theta_{0}^{2}}{Y+(\varepsilon / 3)}-\frac{2+d \phi / d Y}{(2+d \phi / \partial Y)} \frac{\psi_{0}^{2}}{4}=0
\end{aligned}
$$

where Eq. (42) has been employed.

As it was explained in the preceding subsection, Egs. (49) through (51) must satisfy at $Y=1$ the particular combinations of $\psi_{\infty}^{2}, \theta_{\infty}^{2}$ and $(d \phi / d y)$ which satisty Egs. (33), and the condtion of $\phi=2$.

Note that the terms of $O(\varepsilon)$ appear in Egs. (49) through (5]) becuse of the, trarsition layer. outex layor does not contain them as seen in Eqs. (3o) through (32). Therefore, to be consistent with the first arder solution, it is only required that Eqs. (49) through (51) satisfy the boundary conditions at $Y=I$ to $O(\varepsilon)$.

The quentities $\psi_{\infty}^{2}$ and $\theta_{\infty}^{2}$ appearing in Egs. (49) through (51) are fixst replaced in terms of (d申/AY) by the use of Egs. (33). Eguations (49) through (51) are then manipulated into the following set of equations.

$$
\begin{gathered}
\frac{d \phi}{d Y}=T^{2}-2 \\
\Gamma^{3}-\frac{(d \phi / d Y)_{\infty}}{2\left[(2+d \phi / d Y)_{\infty}(Y+\varepsilon / 3)\right]^{I / 2}}-2 \Gamma-\frac{(d \phi / d Y)_{\infty}(2+d \phi / d Y)_{\infty}^{Y / 2}}{2(Y+\varepsilon / 3)^{3 / 2}}=0
\end{gathered}
$$




$$
\begin{aligned}
& \theta_{0}^{2}=\frac{(6 \pi)^{1 / 2}}{2}\left[\frac{2+a_{0} / \partial Y}{(2+a \phi / d Y)_{\infty}}\right]^{1 / 2}(Y+\varepsilon / 3)^{1 / 2}\left(1-\frac{\varepsilon}{2} \frac{d \phi}{\partial Y}\right) \\
& \psi_{0}^{2}=4 \frac{(2+a \phi / \partial Y)_{\omega}}{2+d \phi / d X} \frac{\theta_{0}^{2}}{Y+\varepsilon / 3}
\end{aligned}
$$

With the boundary condition that,

$$
\phi_{0}(1)=2
$$

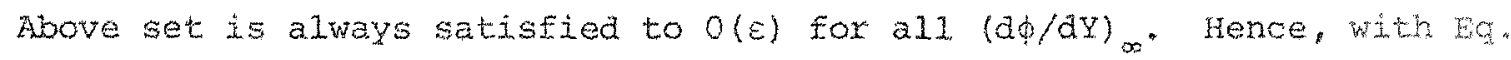

(56) satisfied by Eq. (52), all boundary conditions at $Y=1$ are now satisfied by Eqs. (52) through (56).

The parameter $\varepsilon$ is determined through the subsequent matching with the inner solution.

The standari solution, of course, exists for the cubic equation, Eq. (53). Equation (52) is integrated numerically to satisfy Eq. (56).

\section{Inner Layex}

The stretched inner variable is that defined by $\mathrm{Eq}$. (43).

To be consistent with the outex-transition layer, the terms of $O(E)$ will be retained. Hence, the dependent variables are expanded as,

$$
\begin{aligned}
& \phi(\alpha, \xi)=\alpha(\xi)+0\left(\alpha^{3}\right) \\
& \psi^{2}(\alpha, \xi)=\psi_{0}(\xi)+o\left(\alpha^{2}\right) \\
& \theta^{2}(\alpha, \xi)=\theta_{0}^{2}(\xi)+o\left(\alpha^{2}\right)
\end{aligned}
$$


Again, the terms of $O(a)$ will be consistently retained for the Eirst order in view of Egs. (57). A substitution of the above series into Eqs* (23) through (25), and the subsequent manipulation result, to first order, in the following equations.

$$
\begin{aligned}
& \frac{a^{2} \Phi_{0}}{d \xi^{2}}=\frac{2}{(6 \pi)^{1 / 2}} \frac{d \Psi_{0} \theta_{0}}{d \xi} \\
& \frac{d^{2} \psi_{0}^{2}}{d \xi^{2}}=\frac{4}{(6 \pi)^{1 / 2}}\left\{\Psi_{0} \psi_{0} \frac{\partial \Phi}{d \xi}+\frac{1}{4}\left(\frac{d \phi}{d Y}\right)_{\infty}\left[\frac{2 \varepsilon+d \Phi / d \xi}{(2+d \phi / d Y)}+3 \Psi_{\infty}^{2}\right\}\right. \\
& \frac{d^{2} \theta_{0}^{2}}{d \xi^{2}}=\frac{1}{(6 \pi)^{1 / 2}}\left(\frac{d \phi}{d Y}\right)\left[3 \theta_{\infty}^{2}-\frac{2 \varepsilon+a \phi / d \xi}{(2+d \phi / d Y)} \frac{\rho}{4}\right]
\end{aligned}
$$

In deriving the above equations, $\psi_{\infty}^{2}$ and $\theta_{\infty}^{2}$ have been replaced in terms of $(\mathrm{d} \phi / \mathrm{dY})_{\text {o }}$ by the use of Eqs. (33). Also, Eq. (42) has been employed.

Equations (58) through (60) must satisfy the wall boundary conditions of Eqs. (27) at $\xi=0$. The other set of the boundary conditions are determined through the matching to be described below.

\section{Matching}

The outer-transition layer and the inner layer will now be matched to first order. From the matching, the parameter $\varepsilon$ and the remaining boundary conditions for Egs. (58) through (60) will be determined for a chosen (d申/ar) ${ }_{\infty}$ Beginning with Eqs. (48), the one term inner expansions of the one term outer expansions $(18)$ become, as $Y \rightarrow 0$, 


$$
\begin{aligned}
& \phi(Y)=\phi_{0}(0)+\left(\frac{d \phi_{0}}{d Y}\right)_{0}(\varepsilon \alpha) \xi, \\
& \psi^{2}(Y)=\psi_{0}^{2}(0), \quad \theta^{2}(Y)=e_{0}^{2}(0),
\end{aligned}
$$

where it has been anticipated that $\phi_{0}(0)$ will vanish.

Next, using Eqs. (57), the one term outer expansions of the one term innex expansions become, as $\xi \rightarrow \infty$,

$$
\begin{aligned}
& \phi(Y)=\alpha \lim _{\xi \rightarrow \infty} \Phi_{0}(\xi) \\
& \psi^{2}(Y)=\Psi_{0}^{2}(\infty), \quad \theta^{2}(X)=\theta_{0}^{2}(\infty) .
\end{aligned}
$$

Matching the terms of Egs. (62) to those of Eqs. (61), these result,

$$
\begin{array}{ll}
\phi_{0}(0)=0, & \lim _{\xi \rightarrow \infty} \frac{d \Phi_{0}}{d \xi_{E}}=\varepsilon\left(\frac{d \phi_{0}}{d Y}\right)_{W}, \\
\Psi_{0}^{2}(\infty)=\psi_{0}^{2}(0), & \theta_{0}^{2}(\infty)=\theta_{0}^{2}(0) .
\end{array}
$$

The subscript wenotes $Y=0$.

. The first of Eqs. (63) is the additional boundary condition which the outertransition layer equations must satisfy in order to accomodate the matching. Therefore, for a given homologous boundary condition at $Y=1$ represerted by $(\mathrm{d} \phi / \mathrm{dX})_{\infty}$ there exists a particular value of $\varepsilon$ for which $\phi_{0}(0)=0$ can be satisfied. Once the first order outer solution is obtained, the matching first order inner solution is obtained by numerically integrating Eqs. (58) through (60) to satisfy the wall boundary conditions and the remainder of Eqs. $(63)$. 


\section{DISCUSSION OF SOLUTIONS OBTAINED}

The structure of the Couette flow has been described and discussed in Section IV in terms of certain singular behavior of the governing equations. The solutions of the equations obtained in the preceding section will be aiscussed herein.

Figures 3 and 4 show the typical matching between the inner and the outertransition layers for the mean velocity $u_{0}$, turbulence energy $\left\langle U_{K} U_{K}\right\rangle$, Reynolds stress $\langle U V\rangle$, and the stream-wise component of the turbulence energy $\left\langle U^{2}\right\rangle$. These values have been obtained from the first order solutions of $\phi, \psi^{2}$, and $\theta^{2}$ as"

$$
\begin{aligned}
& \frac{u_{0}}{u_{\infty}}=\frac{\langle u\rangle}{\langle u\rangle_{\infty}}=\frac{1}{u_{O^{\infty}}} \int f u d \vec{U}=\frac{\phi}{2} \\
& \frac{U_{K} U_{K}>}{u^{2}}=\frac{1}{u^{2}} \int f U_{K} U_{K} d \vec{U}=\theta^{2}+\frac{\psi^{2}}{4} \\
& \frac{\langle U V\rangle}{u^{*}}=\frac{1}{u^{*}} \int \text { fUVdU }=\frac{1}{(6 \pi)^{1 / 2}} \psi \theta \\
& \frac{\left\langle\mathrm{u}^{2}>\right.}{u^{*^{2}}}=\frac{1}{u^{*}} \int f \mathrm{U}^{2} \mathrm{du}=\frac{\theta^{2}}{3}+\frac{\psi^{2}}{4}
\end{aligned}
$$

Figure 5 shows the typical mean velocity profiles computed in the present study. Also show are the profiles experimentally obtained by Reichardt ${ }^{(4)}$ and Robertson. (5) The comparison between these experimental results and the present theoretical values all at the Reynolds number of approximately $10^{4}$ shows a satisfactory agreement. 
There have been several empirical and phenomenological theories proposed for the mean velocity profiles of couette flow in the past. Many of these are mentioned in Robertson's work. $(5,6)$ Also, one of the more recent ones is that given by Lundgren. (19) No attempt, however, is made here to compare the present profile with those obtained by the phenomenological theories.

Figure 6 shows the typical profiles of the turbulence energy, Retmolds stress, and the $x$-component of the turbulence energy. Also, the more detailed near-surface values of $\left\langle U^{2}\right\rangle$ are plotted on Fig. 7 since the experimental measurement of $\left\langle\mathrm{U}^{2}\right\rangle$ was taken by Robertson. (6)

As it is expected from the singular behavior of the governing equations discussed in Section IV, 〈UV> remains practically constant until the viscosity effect becomes manifest in the transition layer. The influence of the viscosity on $\left\langle\mathrm{U}_{K} \mathrm{U}_{K}>\right.$ becomes manifest only with the arrival of the inner layer as it was evident in the discussion of Section IV.

The present theory is seen to correctly predict the order of magnitude and the general behavior, with respect of $y$, of $\left\langle\mathrm{U}^{2}\right\rangle$ measured by Robextson. (6) $\left\langle\mathrm{U}^{2}\right\rangle$ increases near the wall. This is evidently caused by the eddy stretching in the $\mathrm{x}$ dixection near the wall.

The coefficient of friction, $C_{f}$, computed in the present theory and those experimentally obtained by Reichardt ${ }^{(4)}$ and Robertson $(5,6)$ are shown in Fig. 8 as the functions of the Reynolds number. Also shown is the empirical curve which Robertson found to be the best fit to his experimental points and to the experimental points obtained in the circuiar couette flow. (20) since a secondary flow can easily exist in a circular couette flow, the applicability of the circular couette flow results to the plane couette flow studies is somewhat in aoubt.

Figure 8 shows that the present theory gives the values of $c_{f}$ which are bem tween those experimentally obtained by Reichaxdt ${ }^{(4)}$ and Robertson $(5,6)$ for the. plane couette Elow. 


\section{CONCLUDING REMRRKS}

The simplified statistical theory developed by the present author earlier has been made self-containing for the shear flows along solid boundaries. This has been accomplished in part by formulating the expression for the contribution of the small eddies to the observabie properties from the available stochastic analyses $(16,17)$ of the Navier-Stokes euqation. During the course of this formum lation, the comparison of the present theory, developed from the generalized Brownian motion, $(7,8,9)$ with the stochastic theory based on the wavier-stokes equation, $(16,17)$ was made. The mutual consistency of the two theories was shown.

The present theory naturally divides the flow region along a wall into the three regions of the outer, transition, and the inner layers. The contribution of the small edaies to the observable properties through viscosity is negligibie in the outer layer whereas its contribution to the mean shear is of first order in the transition layer. In the inner layer, all viscosity effects are of first order.

The comparison of the couette flow solutions obtained with the avajable experimental results shoved that the present theory describes the rather detailed structure of the flow field, such as $\left\langle u^{2}\right\rangle$, satisfactorily in addition to coxrectly preacting the mean velocity profile and the surface shear stress.

It has been shown that the present simplified statistical theory can be employed to analyze the real wall shear flows tractably. 


\title{
ACKNOWLEDGEMENTS
}

This work was supported by the National Aeronautios and space Administxation NGR 14-012-012. The encouragement given by Mr. G. Goodwin and Dr. P. Nachtsheim of Ames Research Center is greatly appreciated. The author is also grateful to Mr. T. J. Marcisz, a graduate student in the Enexgy Engineering Department. Eor his help in obtaining the numerical results of this paper.

\author{
REFERENCES
}

1. P. A. I.ibby, ARS J. 32, 388 (1962).

2. D. E. Rosner, Chem. Eng. Sci., 19, 1 (1964).

3. W. H. Webb and I. A. Hxomas, AIAA J., 3, 826 (1965).

4. V. H. Reichardt, Z. Angew, Math. Mech., 36, 26 (1956).

5. I. M. Robertson, in Proceedings of the 6 th Midwest Fluid Mechanics Conference (University of Texas Press, Austin, Texas, 1959) p. 169.

6. J. M. Robertson, H. F. Johnson, Journal of the Engineering Mechanics Division, Proceedings of the American Society of Civil Engineers, December, 1970, $p .117$.

7. P. M. Chung, AIAA J., 7, 1982 (1969).

8. P. M. Chung, Phys. FIuids, 13, 1153 (1970).

9. P. M. Chung, AIAA Preprint No. 70-722 (1970); also to be published in AIAR J. (1971).

10. T. C. Chawla, Singular-Perturbation Theory of Turbulent Boundary Iayers with Negligible Wall stress, Ph. D. Thesis, Department of Nuclear Engineering. penn. State University, State college, Pa., June (1969).

11. C. Y. Iiu and $\mathrm{L}$. Lees, in Rarefied Gas Dynamics, L. Talbot, Ed. (Academic Press Inc., New York, 1961), p. 391.

12. G. K. Batchelor, The Theory of Homogeneous Turbulence, (Cambridge University Press, New York, 1953), Chap. 6.

13. I. O. Hinze, Turbulence, (McGraw-Hill Book Company, New York, 1959), Chaps. 3 and 4 . 
14. J. Laufer, NACA Tech. Repts., 2174, (1954).

15. P. S. Klebanoff and Z, W. DiehI, J. Research NBS, 50, (1953).

16. T. S. Lundgren, Phys. Fluids 12, 969 (1967).

17. R. Fox, Solution for the Correlation Functions in a Homogeneous Isotropic Incompressible Turbulent Field, Sandia Laboratory Report SC-RR-70-912, AIbuquerque, N. M., February, 1971. Also to be published in Phys. Fluids (1971).

18. M. D. Van Dyke, Perturbation Methods in Fluid Mechanics, Academic Press, New York, (1965).

19. T. S. Lundgren, Phys. Fluids, 14, 225 (1971).

20. M. Couette, Ann. De Chemie et de Physique, Ser. 6, 21, 433 (1890). 
$-35-$
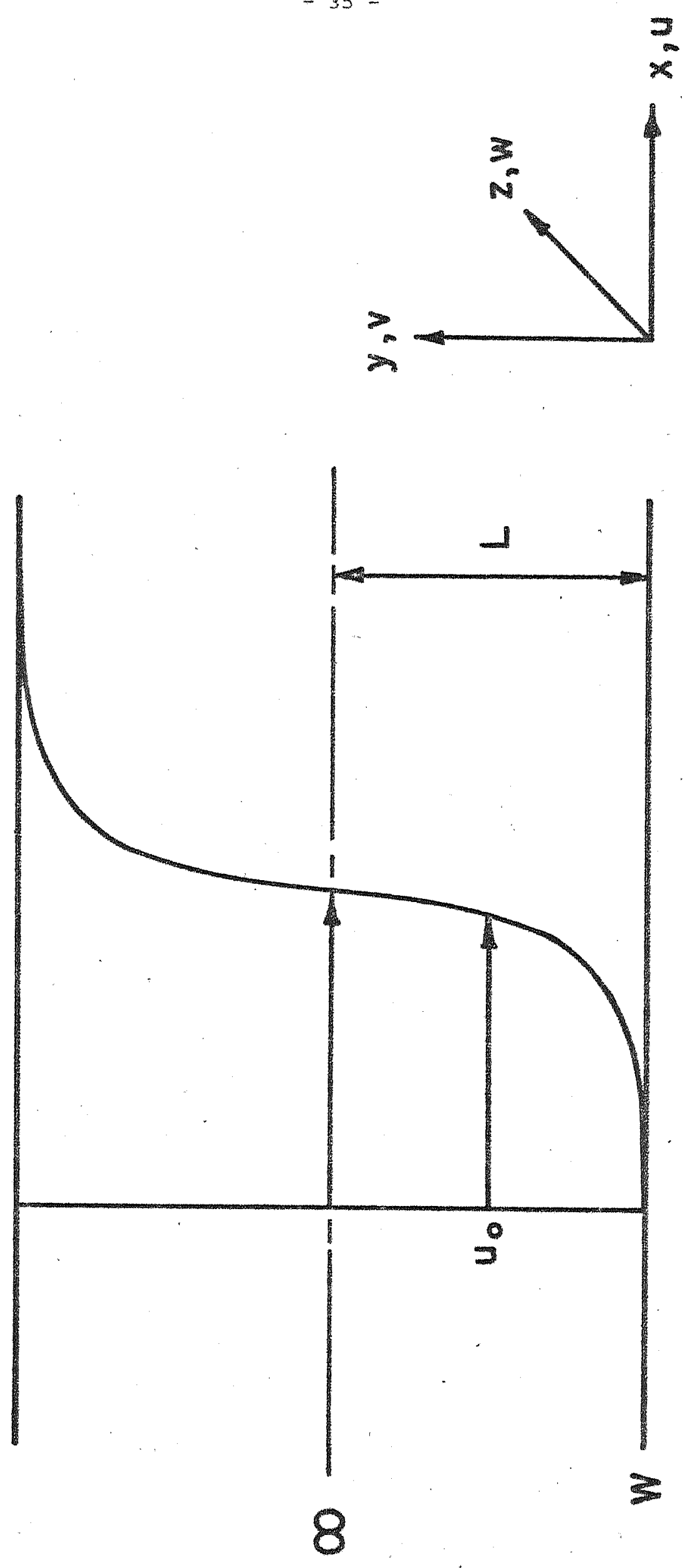


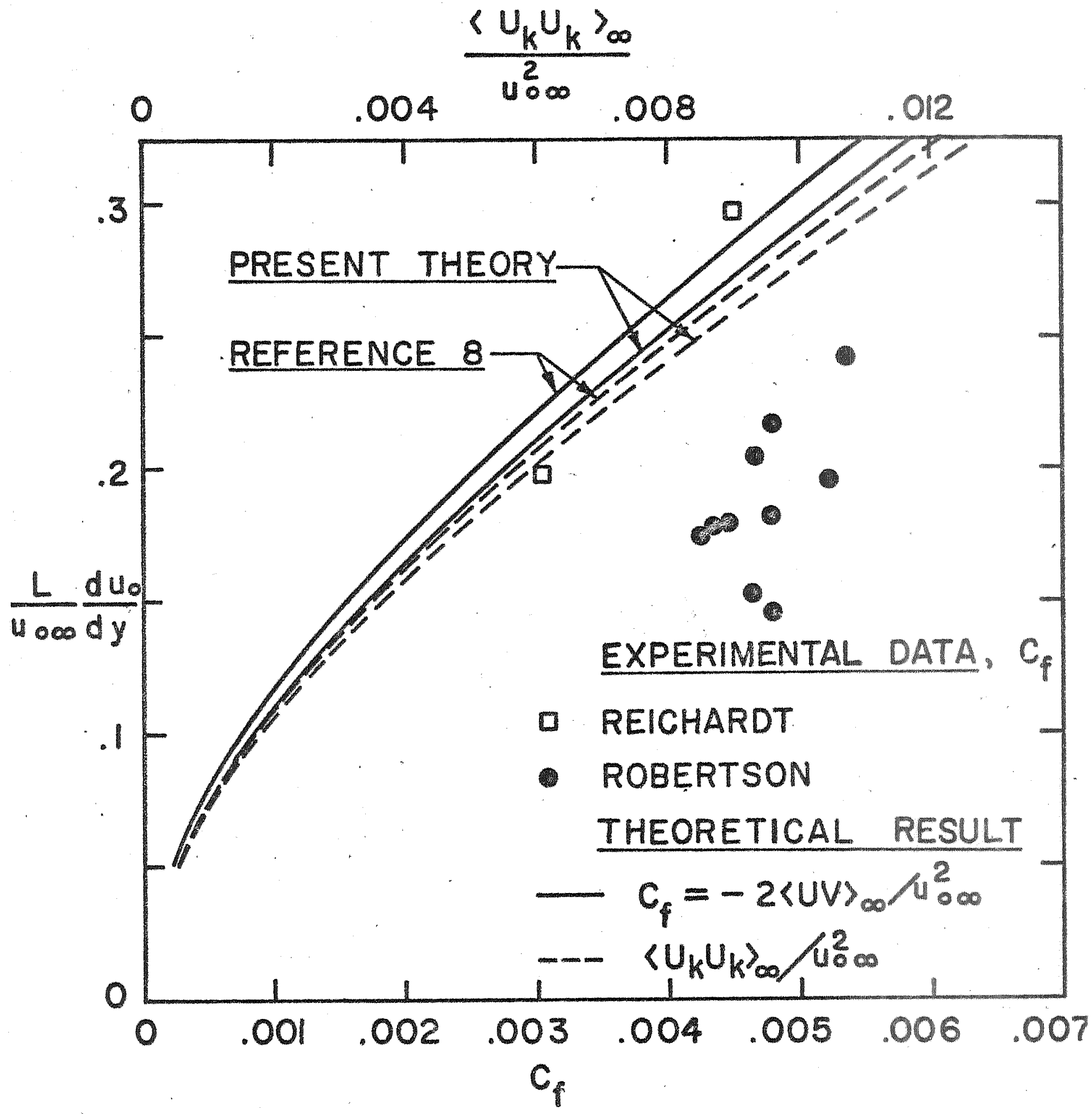

FIGURE 2: REYNOLDS STRESS AND TURBULENCE FNERGY 


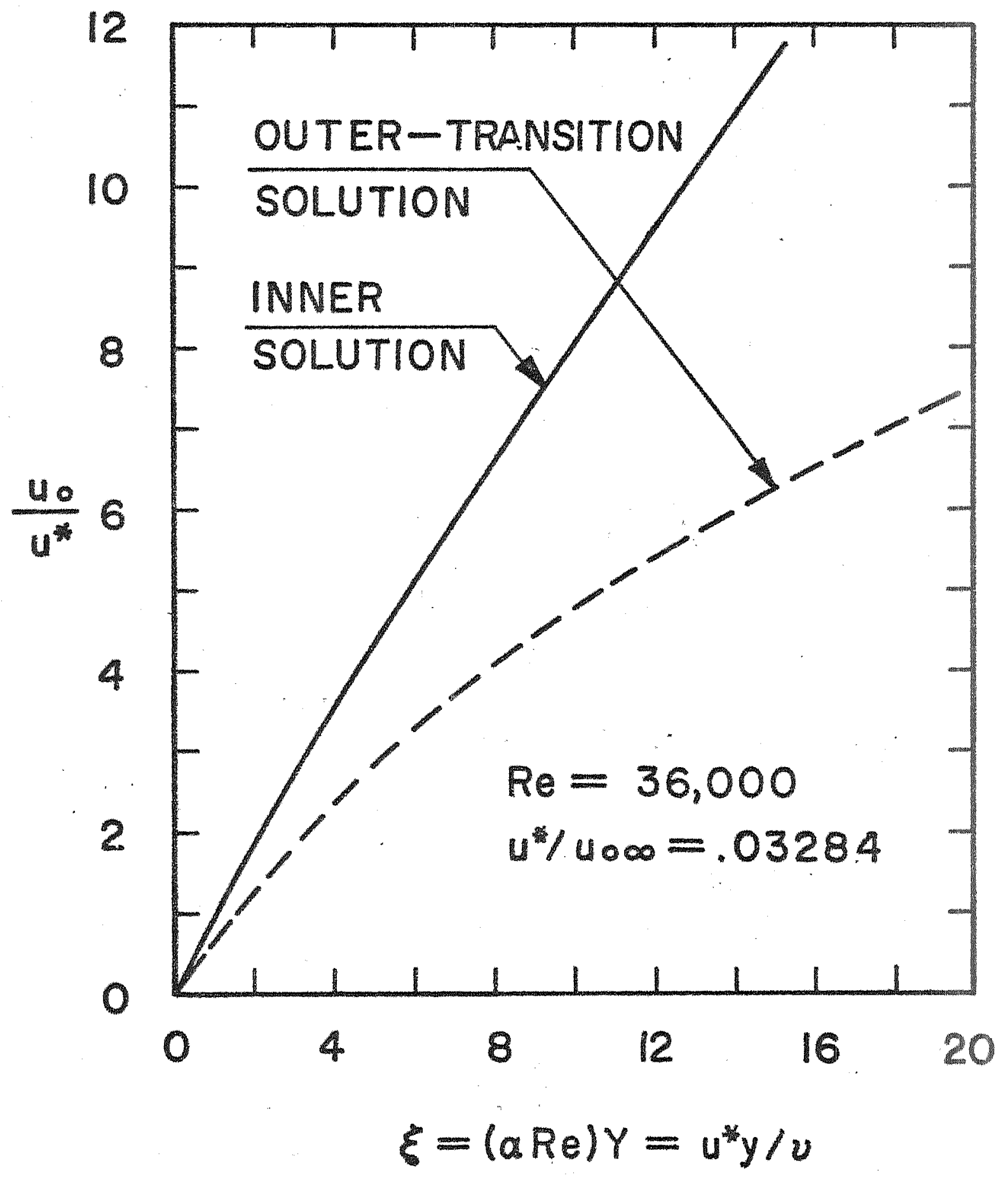




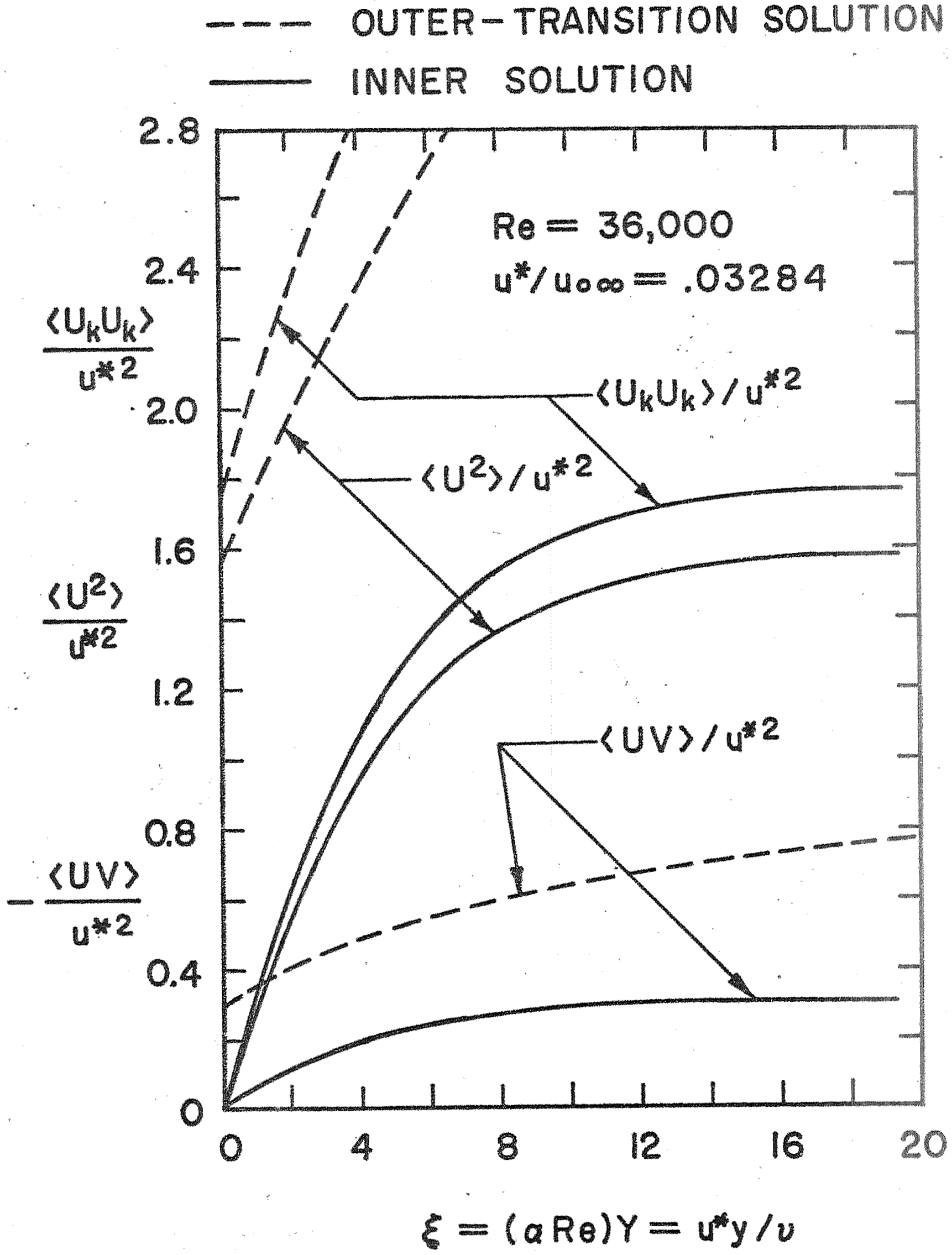

FTGURE 4: TYPTCZI ASTMETOTIC MATCHTIV BETVEEN 


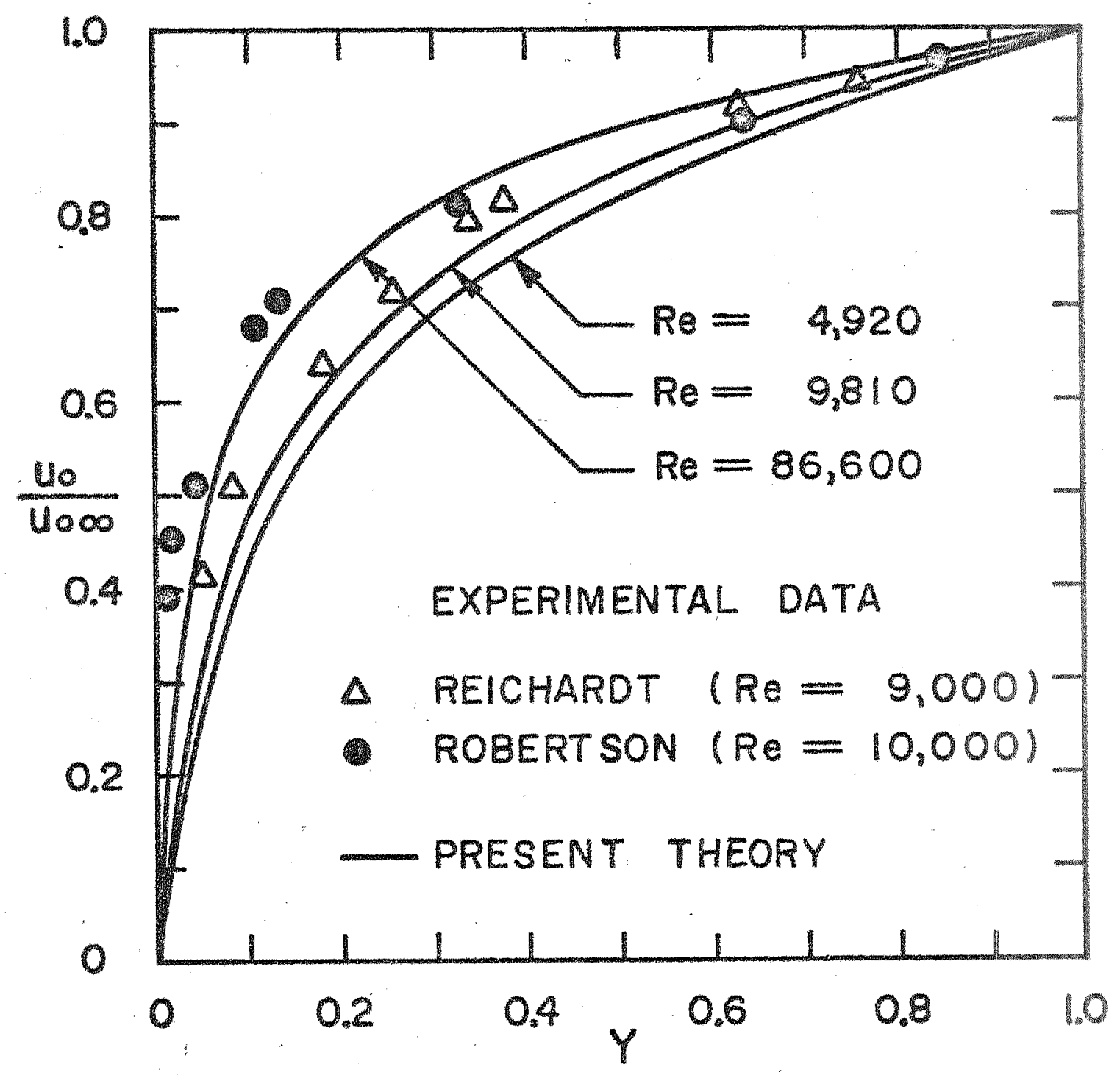

FTGURE S: MEAN VESOCITY PROFILES 


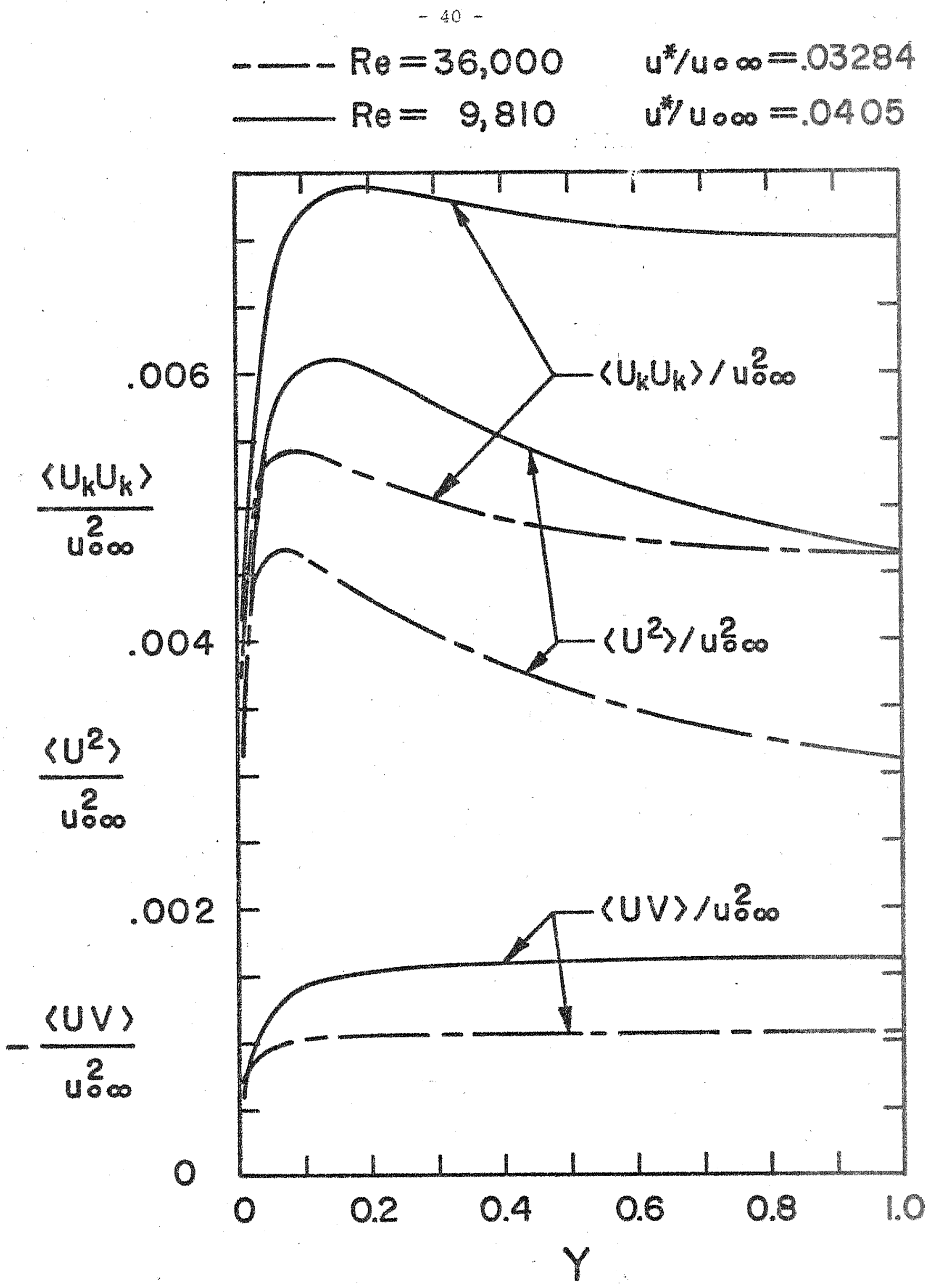

PIGURE 6: TYPTCAI TURBULENCE STRUCTURE OF COUETTE FLOW 


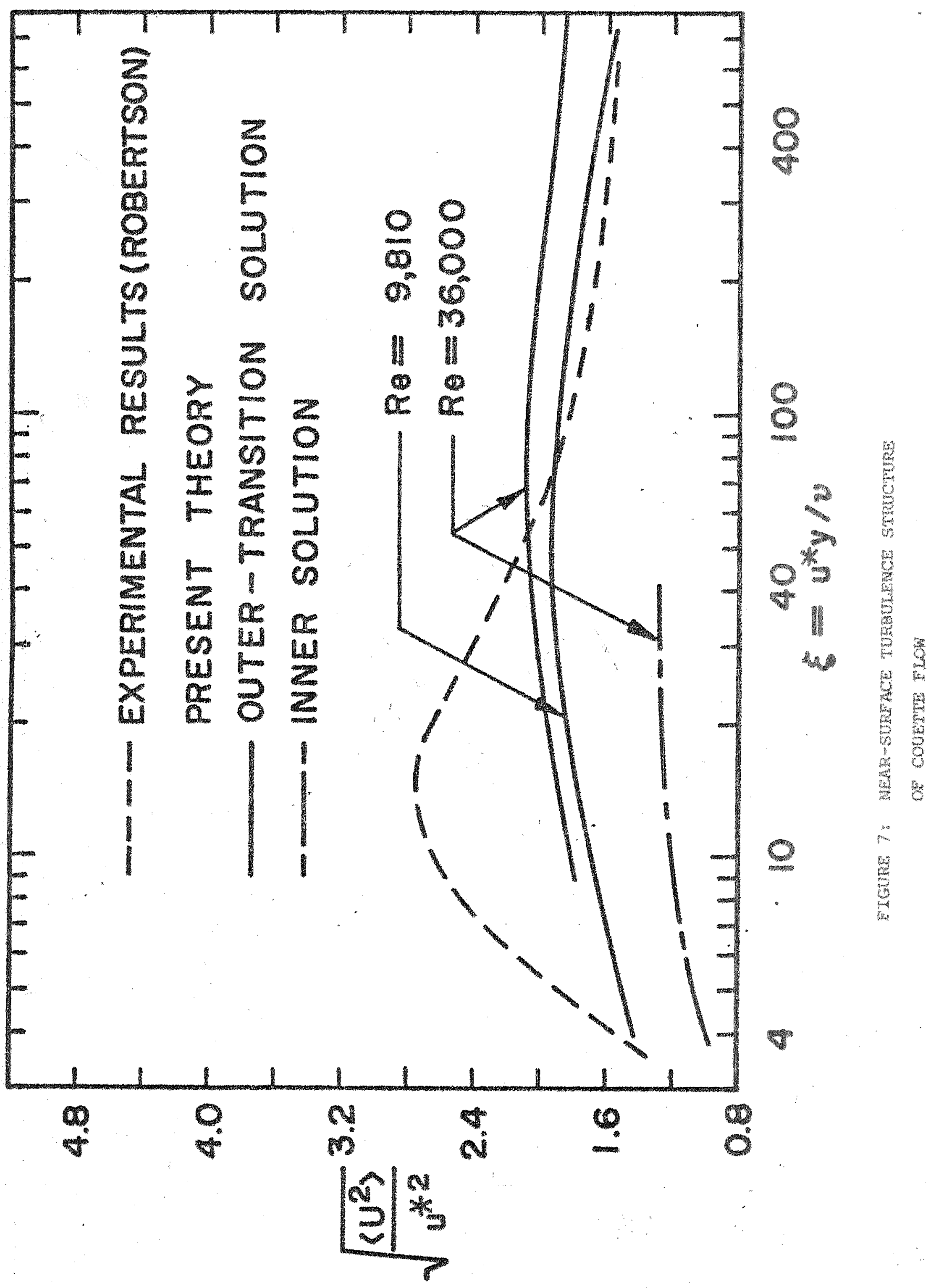




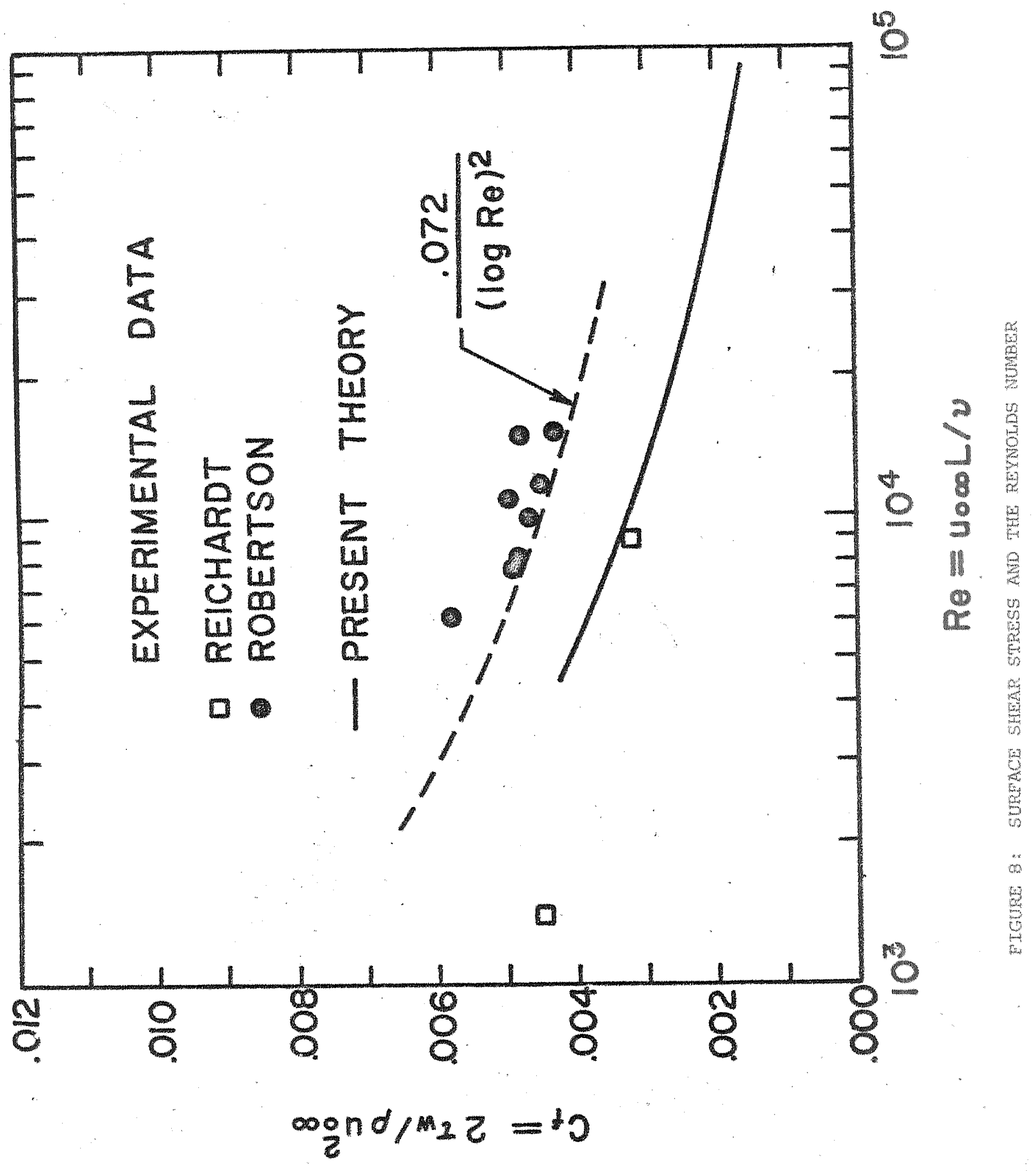

